Revue d'histoire de l'enfance " irrégulière »

Le Temps de l'histoire

$10 \mid 2008$

La prostitution des mineur(e)s au $X^{\mathrm{e}}$ siècle

\title{
Bruno Carlier, Sauvageons des villes, sauvageons aux champs. Les prises en charge des enfants délinquants et abandonnés dans la Loire (1850-1950)
}

Pascale Quincy-Lefebvre

\section{(2) OpenEdition \\ Journals}

Édition électronique

URL : http://journals.openedition.org/rhei/2982

DOI : 10.4000/rhei.2982

ISBN : 978-2-7535-1649-6

ISSN : $1777-540 \mathrm{X}$

Éditeur

Presses universitaires de Rennes

Édition imprimée

Date de publication : 1 octobre 2008

Pagination : 208-210

ISSN : $1287-2431$

Référence électronique

Pascale Quincy-Lefebvre, «Bruno Carlier, Sauvageons des villes, sauvageons aux champs. Les prises en charge des enfants délinquants et abandonnés dans la Loire (1850-1950)", Revue d'histoire de l'enfance " irrégulière » [En ligne], 10 | 2008, mis en ligne le 18 novembre 2009, consulté le 04 décembre 2020. URL : http://journals.openedition.org/rhei/2982 ; DOI : https://doi.org/10.4000/rhei.2982 


\section{Bruno Carlier}

Sauvageons des villes, sauvageons aux champs. Les prises en charge

des enfants délinquants et abandonnés dans la Loire (I850-1950)

Saint-Étienne, Publications de l'université de Saint-Étienne, 2006, 460 p.

ISBN 978-2862724225

L'ouvrage de Bruno Carlier reprend le contenu d'une thèse fort riche soutenue en 2004 à l'université Louis Lumière de Lyon II. L'auteur, actuellement enseignant dans le département de la Loire, membre du Centre de recherche en histoire (CERHI) de l'université Jean Monnet de Saint-Étienne, a su mettre à profit son excellente connaissance du milieu local pour produire une recherche pointue sur l'histoire de la protection de l'enfance et de la jeunesse. Un des intérêts de cette recherche réside dans l'ampleur de l'enquête archivistique sur un siècle d'histoire. C'est ce travail de terrain qui permet à Bruno Carlier de montrer l'importance des acteurs locaux et du contexte local, ici celui d'une grande région industrielle, pour analyser des processus et évolutions permettant de mieux comprendre l'émergence et la structuration d'un champ de la protection de l'enfance en France. La richesse et la difficulté de l'entreprise tiennent à la démarche adoptée : par l'étude des prises en charge des enfants délinquants et abandonnés, ne pas focaliser la recherche sur la fabrication par une administration d'une " clientèle » mais historiciser la question plus globale des prises en charge de l'enfance irrégulière en s'attardant sur les relations complexes entre justice et assistance, initiatives publiques, actions privées pour mieux étudier, à l'échelle locale, l'intégration par les acteurs d'une réflexion sur "l'enfance en danger ", l'émergence de ce qui pourrait être qualifié de réseau. La tâche n'est pas aisée puisqu'il s'agit de travailler sur "l'enfance en danger ", une catégorie historiquement construite à partir de logiques d'intervention différentes (justice, assistance), susceptible de relever de l'action publique ou privée. Les développements sont riches. On peut seulement regretter que l'éclatement des présentations ne soit pas davantage assorti d'une étude plus resserrée de ce qui justifie de rassembler ces populations d'enfants « qui vont mal ».

Trois parties structurent l'ouvrage. Leur organisation vise à dégager un certain nombre d'évolutions propres à donner une histoire à un secteur, celui de

Pascale Quincy-Lefebvre / p. 208 à 210 
l'intervention sociale aux fils étrangement tissés avec son passé. La première partie privilégie l'histoire des prises en charge publiques : Assistance publique et Justice avant la deuxième guerre mondiale à partir de logiques d'intervention qui caractérisent l'État de droit et qui distinguent le délinquant de l'assisté. Les pratiques judiciaires évoluent, et il faut saluer ici le gros travail statistique de l'auteur qui, même s'il demanderait à être précisé sur un plan méthodologique, apporte de très précieuses informations sur le temps long. Essentiellement centrée sur un long XIXème siècle, cette partie s'attarde sur le cadre légal, relève la place des individus et de l'action associative dans l'inflexion des modèles d'action publique. À l'image des travaux pionniers de Michel Chauvière ou des études de David Niget sur Angers ou de Dominique Dessertine pour Lyon, l'analyse du fonctionnement de la justice des mineurs dans la Loire invite à voir dans les années 1930, une décennie qui pèse lourd, une idée davantage développée dans la troisième partie.

L’organisation de la seconde partie centrée sur le XIXème siècle est moins convaincante. Certes, l'auteur entend démontrer le rôle fondamental de l'initiative privée dans le développement d'une prise en charge « éducative " de l'enfance à problème en insistant sur le rôle des acteurs par rapport aux institutions. L'approche monographique est privilégiée dans l'étude des prises en charge. Mais l'importance accordée à la colonie agricole et industrielle de SaintGenest-Lerpt peut déconcerter le lecteur alors plongé dans une histoire particulière qui, par son ampleur, s'écarte du projet initial. L'auteur justifie ses choix par ses sources et l'intérêt de cette institution, émanation de Cîteaux et du père Rey, dominée par la figure du père Cœur et pour laquelle l'auteur pose la question de l'influence de la pédagogie de Don Bosco lorsqu'il évoque la " conception intéressante et moderne de la pédagogie ». Une lecture plus en creux de la question aurait peut être permis d'analyser les limites de la mobilisation des acteurs privés, laïques avant les années 1930.

Par sa structuration, la troisième partie renoue davantage avec le beau projet de Bruno Carlier. L'auteur étudie avec beaucoup de finesse le processus d'hybridation entre public et privé qui caractérise la structuration du secteur de l'enfance en danger au XXème siècle. Le département de la Loire est remarquable par le dynamisme de ses acteurs publics et privés à partir de l'entredeux-guerres. Tout en évoquant les réorganisations institutionnelles de la Libé- 
ration, les forces nouvelles dont dispose le secteur pour se développer, Bruno Carlier met en évidence la filiation entre la volonté d'unité de la Fédération des œuvres de l'enfance, sa capacité à accueillir l'innovation par le biais de la professionnalisation très progressive des acteurs et la structuration propre à un modèle national de prise en charge de l'enfance en danger à partir des grands textes de Vichy et de la Libération.

La précocité de l'articulation raisonnée entre actions publiques et privées est remarquable dans une région qui n'est pas dominée par une grande métropole à l'exemple de la Seine ou du Rhône. La proximité de Lyon ne peut néanmoins pas être ignorée, ni le poids qu’a pu avoir chez les élites, la représentation des particularités économiques et sociales d'une région anciennement industrielle comme le bassin de Saint Étienne. Pour autant, l'intérêt de l'étude de Bruno Carlier est également de montrer la grande fragilité des constructions de l'époque et le nombre limité d'acteurs engagés. Les transformations des années 1930 reposent sur l'implication de quelques individualités, l'avocate féministe Simone Levaillant, le substitut Mailhol, l'assistante sociale Marinette Heurtier, le pédiatre Charles Beutter. Le rôle de l'inspecteur de l'Assistance publique François Leboulanger est également souligné.

À partir d'exemples précis et en soulignant les tournants des années 18801890 et des années 1930, Bruno Carlier confirme le rôle des relais locaux (pour mettre sur pieds un comité de patronage, une clinique médico-pédagogique), le décalage entre les principes d'une loi (par exemple l'introduction de la liberté surveillée dans la loi de 1912) et les pratiques. Le lien est fait avec une tradition locale philanthropique et paternaliste, le poids de la " vocation religieuse " mais c'est avec l'incorporation d'un nouveau "souci de l'enfant " que l'action individuelle peut s'insérer dans un réseau et gagner en efficacité au travers d'un modèle d'intervention sociale encore " artisanal ». Avec la disparition de la figure omnipotente de Marinette Heurtier, mais aussi la consolidation d'un système dans un contexte de croissance économique et de développement de politiques publiques, les années 1950 marquent non seulement une rupture générationnelle mais signalent l'entrée de l'histoire de la protection de l'enfance dans une autre dimension.

Pascale Quincy-Lefebvre 